Bundesgesundheitsbl 2019 · 62:114-117 https://doi.org/10.1007/s00103-018-2849-1 Online publiziert: 12. November 2018 (c) Springer-Verlag GmbH Deutschland, ein Teil von Springer Nature 2018

Bekanntmachung des Umweltbundesamtes

\title{
Gesundheitliche Bewertung von 1,2-Dichlorethan (1,2-DCE) in der Innenraumluft
}

\author{
Mitteilung des Ausschusses für \\ Innenraumrichtwerte (AIR)
}

\section{Physikalische Eigenschaften und Verwendung}

1,2-Dichlorethan (1,2-DCE) ist im europäischen Gefahrstoffrecht als wahrscheinlich krebserzeugend beim Menschen (Kategorie 1 B) eingestuft [1] und ist ein zulassungspflichtiger Stoff gemäß Anhang XIV der REACH-Verordnung [2]. Aktuelle quantitative Risikoabschätzungen liegen vom Ausschuss für Risikobewertung der Europäischen Chemikalienbehörde (ECHA-RAC) [3] und vom Ausschuss für Gefahrstoffe (AGS) vor [4]. Zudem gibt es eine aktuelle Bewertung von der kanadischen Gesundheitsbehörde [5] und eine umfangreiche Bewertung von der US-amerikanischen Agentur für Toxische Substanzen und die Registrierung von Krankheiten (ATSDR) [6].

\section{Stoffidentifizierung}

$\begin{array}{ll}\begin{array}{l}\text { Systematischer Name } \\ \text { Synonyme }\end{array} & \begin{array}{l}\text { 1,2-Dichlorethan } \\ \text { Chlorethylen, } \\ \text { Ethylendichlorid, } \\ (1,2-) \text { DCE, EDC }\end{array} \\ \text { CLP-Index-Nr. } & 602-012-00-7 \\ \text { EG-Nr. } & 203-458-1 \\ \text { CAS-Nr. } & 107-06-2 \\ \text { Summenformel } & \mathrm{C} 2 \mathrm{H} 4 \mathrm{Cl} 2 \\ \text { Strukturformel } & \mathrm{Cl}\end{array}$

\begin{tabular}{ll} 
Molekulargewicht & $98,96 \mathrm{~g} / \mathrm{mol}$ \\
Schmelzpunkt & $-36{ }^{\circ} \mathrm{C}$ bei $101,3 \mathrm{kPa}$ \\
Siedepunkt & $83,6^{\circ} \mathrm{C}$ bei $101,3 \mathrm{kPa}$ \\
Dampfdruck & $10,25 \mathrm{kPa}$ bei $25^{\circ} \mathrm{C}$ \\
Wasserlöslichkeit & $7,9 \mathrm{~g} / \mathrm{l}$ bei $25^{\circ} \mathrm{C}$ \\
log Poctanolwasser: & $1,45 \mathrm{bei} 20^{\circ} \mathrm{C}$ \\
\hline $\begin{array}{l}\text { Umrechnungsfak- } \\
\text { tor }(\text { bei } 1013 \mathrm{hPa},\end{array}$ & $1 \mathrm{ml} / \mathrm{m}^{3}=4,113 \mathrm{mg} / \mathrm{m}^{3}=0,243 \mathrm{ml} / \mathrm{m}^{3}$ \\
$\left.25^{\circ} \mathrm{C}\right)$ &
\end{tabular}

1,2-DCE ist eine farblose, ölige, leicht entzündbare Flüssigkeit mit chloroformartigem Geruch [7]. 1,2-DCE wird heutzutage vorwiegend industriell genutzt und entsteht als Zwischenprodukt bei der Herstellung von Vinylchlorid, Trichlorethan, Tri- \& Tetrachlorethen oder Ethylenoxid [4, 6]. 1,2-DCE ist unter REACH als Prozess- und Extraktionslösemittel für industrielle Prozesse zugelassen worden [8]. Darüber hinaus wurde im Registrierungsdossier nur die Nutzung als Laborchemikalie genannt [9].

Früher war 1,2-DCE Bestandteil vieler Antiklopfmittel für Benzin, wurde für die Bleientfernung aus Motorölen, zur Entfettung von Metallen, zum Aufspalten von Ölen, Wachsen, Harzen, Gummis und in chemischen Reinigungen verwendet. Zudem war 1,2-DCE in einigen verbraucherrelevanten Produkten enthalten: Abbeizmittel, Reinigungslösungen, Pestizide, Tapetenkleber, Farben oder Lacke $[4,6]$.

\section{Vorkommen in der Innenraumluft}

Die Arbeitsgemeinschaft Ökologischer Forschungsinstitute (AGÖF) hat im Rahmen eines UBA-Forschungsprojektes (2006 bis 2012) die Ergebnisse der anlassbezogenen Messungen in Innenräumen in einer Datenbank zusammengefasst (VOC Datenbank II). In der Gruppe der halogenierten Kohlenwasserstoffe wurde 1,2DCE am häufigsten bestimmt und wurde in rund $10 \%$ der Räume oberhalb der Bestimmungsgrenze nachgewiesen (- Tab. 1). Das Maximum lag bei 1,8 mg/ $\mathrm{m}^{3}$. Die erreichten Bestimmungsgrenzen (BG) lagen für den größten Teil ( $\geq 75 \%)$ dieser Messungen bei $1 \mu \mathrm{g} / \mathrm{m}^{3}$; die kleinste $B G$ wurde mit $0,5 \mu \mathrm{g} / \mathrm{m}^{3}$ angegeben [10]. In einer Studie aus den USA wurde die Innenraumluft von 126 Häusern auf flüchtige organische Substanzen untersucht. In $13 \%$ der Fälle wurde 1,2-DCE oberhalb der Methodennachweisgrenze von $0,39 \mu \mathrm{g} / \mathrm{m}^{3}$ nachgewiesen. Das Maximum lag bei $8 \mu \mathrm{g} / \mathrm{m}^{3}$ und das 95 . Perzentil bei $1 \mu \mathrm{g} / \mathrm{m}^{3}$ [11].

\section{Toxikokinetik}

Die Toxikokinetik von 1,2-DCE wurde tierexperimentell umfänglich untersucht. Für den Menschen werden weitgehend analoge Verhältnisse bezüglich Aufnahme, Verteilung, Metabolisierung und Elimination angenommen. Das lipophile 1,2-DCE wird gut über die Atemwege, Haut und Magen-Darm-Trakt aufgenommen und anschließend schnell im Kör- 
Tab. 1 1,2-DCE in der Innenraumluft [10]

\begin{tabular}{llll} 
Innenraum & $\boldsymbol{n}$ & $\boldsymbol{n}>\mathrm{BG}[\%]$ & $\mathbf{9 5 .}$ Perzentil $\left[\boldsymbol{\mu g} / \mathrm{m}^{3}\right]$ \\
\hline Nicht spezifiziert & 2061 & 10 & 1 \\
Wohnraum & 472 & 16 & 2 \\
Büroraum & 1022 & 9 & 1 \\
Klassenraum & 312 & 6 & 1
\end{tabular}

Tab. 2 Tumorinzidenzen F344-Ratten-Weibchen nach zweijähriger inhalativer Exposition gegenüber 1,2-DCE (>99\%) [17]

\begin{tabular}{|c|c|c|c|c|c|}
\hline \multicolumn{2}{|l|}{ Effekt } & \multicolumn{4}{|c|}{ Konzentration } \\
\hline Organ & Befund & $0 \mathrm{ml} / \mathrm{m}^{3}$ & $10 \mathrm{ml} / \mathrm{m}^{3}$ & $40 \mathrm{ml} / \mathrm{m}^{3}$ & $160 \mathrm{ml} / \mathrm{m}^{3}$ \\
\hline Unterhaut & Fibrom & 0 & 0 & 1 & $5^{\mathrm{a}}$ \\
\hline \multirow[t]{5}{*}{ Brustdrüse } & Adenom & 3 & 5 & 5 & $11^{\mathrm{a}}$ \\
\hline & Fibroadenom & 4 & 1 & 6 & $13^{\mathrm{a}, \mathrm{c}}$ \\
\hline & Adenokarzinom & 1 & 2 & 0 & 5 \\
\hline & $\begin{array}{l}\text { Adenom + } \\
\text { Fibroadenom }\end{array}$ & 7 & 6 & 11 & $22^{a, c}$ \\
\hline & $\begin{array}{l}\text { Adenom }+ \\
\text { Fibroadenom }+ \\
\text { Adenokarzinom }\end{array}$ & 8 & 8 & 11 & $25^{b}$ \\
\hline \multicolumn{6}{|c|}{$\begin{array}{l}N=50 \text { je Gruppe } \\
\text { a,b signifikant verschieden zur Kontrolle } p \leq 0,05 \text { bzw. } \leq 0,01 \text { Fishers Exakt Test } \\
\text { 'ebenfalls signifikanter Effekt bei den Männchen; } 1 \mathrm{ml} / \mathrm{m}^{3}=4,113 \mathrm{mg} / \mathrm{m}^{3}\end{array}$} \\
\hline
\end{tabular}

per verteilt. In Arbeitsplatzstudien fand man 1,2-DCE auch in der Muttermilch. In tierexperimentellen Studien (Ratten, $153-1999 \mathrm{ml} / \mathrm{m}^{3} \quad\left(629-8222 \mathrm{mg} / \mathrm{m}^{3}\right)$, 5 Stunden am 17. Gestationstag) wurde 1,2-DCE dosisabhängig in Föten nachgewiesen [12].

In Inhalationsversuchen an Ratten stellte sich in Abhängigkeit von der Expositionskonzentration nach zwei Stunden $\left(50 \mathrm{ml} / \mathrm{m}^{3}\left(206 \mathrm{mg} / \mathrm{m}^{3}\right)\right)$ bzw. drei Stunden $\left(250 \mathrm{ml} / \mathrm{m}^{3}\left(1028 \mathrm{mg} / \mathrm{m}^{3}\right)\right)$ ein Konzentrationsgleichgewicht im Blut ein. Die fünffache Expositionskonzentration führte zu 20-30 fachen Konzentrationsanstiegen in verschiedenen Geweben (Fett, Blut, Lunge und Leber) [5].

An männlichen Osborne-Mendel-Ratten wurde die Verteilung und Ausscheidung von C-14 markiertem 1,2-DCE nach oraler Gabe per Schlundsonde (150 mg/ $\mathrm{kg} \mathrm{KG)} \mathrm{mit} \mathrm{der} \mathrm{nach} \mathrm{inhalativer} \mathrm{Expositi-}$ on (6 Stunden, $150 \mathrm{ml} / \mathrm{m}^{3}\left(617 \mathrm{mg} / \mathrm{m}^{3}\right)$ ) verglichen. Die Verteilungsmuster waren, abgesehen vom Vormagen, sehr ähnlich und die Ausscheidung war bei beiden Applikationsarten nach 48 Stunden quasi vollständig. Der Großteil (ca. 85 \%) wurde in Form von Thiodiessigsäure und Thiodiessigsäure Sulfoxid im Urin nachgewiesen. 7-8 \% fanden sich im ausgeatmeten Kohlendioxid, $2 \%$ in den Fezes und $4 \%$ blieben im Körper [13].

Für 1,2-DCE werden zwei verschiedene Abbauwege diskutiert. Zum einen wird es über Cytochrom P450 (CYP2E1) zum reaktiven 2-Chloracetaldehyd oxidiert, das entweder mit NADH zu 2-Chlorethanol reduziert, oder mit Aldehyddehydrogenase $(\mathrm{AlDH}) \mathrm{zu}$ 2-Chloressigsäure 2-Formylmethyl-glutathion verstoffwechselt werden kann. Zum anderen kann 1,2DCE auch direkt mit GSH reagieren. Auf diesem Weg kann ein sehr reaktives Episulfonion-Ion entstehen, das dann weiter zu S-2-Hydroxythylcystein oder S,S'-Ethylenbisglutathion metabolisiert wird $[6,14]$.

Der oxidative Pfad ist in niedrigen Konzentrationen vorherrschend und weist eine hohe Affinität aber mit geringer Kapazität auf, während der Umsetzung mit GSH eine geringe Affinität mit hoher Kapazität zugeschrieben wird [15, 16]. Der Metabolismus scheint bei den oxidiert oder mit Glutathion (GSH) zu Sverschiedenen Spezies und Stämmen ähn- lich zu sein, jedoch mit unterschiedlichen Umsetzungsraten und Sättigungskonzentrationen. Für Osborne-Mendel-Ratten liegen Abschätzungen vor, wonach der oxidative Pfad bei einer Blutkonzentration von $5-10 \mu \mathrm{g} / \mathrm{L}$ gesättigt ist; dies entspricht einer Konzentration von $150 \mathrm{ml} /$ $\mathrm{m}^{3}\left(617 \mathrm{mg} / \mathrm{m}^{3}\right)$ [13].

\section{Bewertung}

Die Kanzerogenität von 1,2-DCE wurde mit Studien inhalativer, oraler oder dermaler Exposition an Nagern untersucht. Nach inhalativer 1,2-DCE-Exposition wurden Brustdrüsen-, Bronchioalveolarund Lebertumore sowie Hämangiosarkome (Leber, Milz) beobachtet [4]. In den aktuellen Risikoabschätzungen des ECHA-RAC [3] und des AGS [4] wurde jeweils die Studie von Nagano et al., 2006 [17] als Schlüsselstudie gewählt. In dieser zweijährigen Inhalationsstudie an F344Ratten traten insbesondere bei den Weibchen dosisabhängig erhöhte Tumorinzidenzen auf (• Tab. 2).

Die Wirkweise von 1,2-DCE, die zu der beobachteten Vielzahl an Tumoren in den verschiedenen Kanzerogenitätsstudien führte, ist noch nicht komplett verstanden. Während 1,2-DCE in vitro mutagen wirkt, wurden in vivo keine mutagenen Effekte beobachtet. Die in vivo 1,2-DCE-induzierten gentoxischen Effekte (DNA-Addukte, -Strangbrüchen, Schwesterchromatidaustausch) in diversen Organen scheinen von der Expositionshöhe und -dauer abzuhängen; für das Zielgewebe (Brustdrüsen) gibt es nur eine Studie mit nur einer Dosis. In diesem Comet-Assay wurden vierfach erhöhte DNA-Addukte festgestellt; jedoch keine anderen gentoxischen Effekte (weibliche F344-Raten, 4 Wochen, 7 Tage/Woche, 6 Stunden/Tag, $200 \mathrm{ml} / \mathrm{m}^{3}$ ) [4].

Der AGS hat 1,2-DCE als ein Kanzerogen mit schwachem gentoxischen Potential beschrieben und ECHA-RAC bewertete 1,2-DCE als ein Kanzerogen, bei dem ein gentoxisches Potential nicht ausgeschlossen werden kann. Beide Ausschüsse verwendeten die Summe der Brustdrüsentumore (Adenom/Fibroadenom/Adenokarzinom) zur Risikobewertung, wobei die Ausgangspunkte für die Risikoextrapolation mit unterschiedlichen Verfahren ermittelt wurden; ECHA- 
RAC hat das T25-Konzept angewendet $\left(\mathrm{T}_{25}=406 \mathrm{mg} / \mathrm{m}^{3}=98,8 \mathrm{ppm}\right)$ und der AGS das Benchmarkdose-Verfahren $\left(\mathrm{BMC}_{10}=205 \mathrm{mg} / \mathrm{m}^{3}=49,74 \mathrm{ppm}\right)[3,4]$.

Welcher metabolische Weg tatsächlich für die Tumorentstehung entscheidend ist, ist noch weitgehend ungeklärt, so werden nicht nur die reaktiven Metabolite beider Metabolismus-Pfade als mögliche Ursache diskutiert, sondern auch das 1,2-DCE selbst $[4,5]$. Da die DCE-abhängige Tumor-Entwicklung noch nicht ausreichend verstanden ist, nutzen sowohl ECHA-RAC als auch AGS das lineare Modell für die Extrapolation in den Niedrigdosisbereich [3, 4]. Der AIR übernimmt diesen Ansatz.

Da das BMD-Verfahren die Exposition-Risiko-Kurve modelliert, bevorzugt der AIR das BMD-Verfahren und verwendet die $\mathrm{BMC}_{10}$ für die Ableitung eines risikobezogenen Leitwertes. Nach Extrapolation auf eine potentielle kontinuierliche Exposition mit dem Faktor 5,6 (7 d/5 $\mathrm{d} \times 24 \mathrm{~h} / 6 \mathrm{~h}$ ) ergibt sich eine $\mathrm{BMC}_{10}$ von $36,6 \mathrm{mg} / \mathrm{m}^{3}$. Ein Risiko von $10^{-6}$ (risikobezogener Leitwert) ist danach bei einer Konzentration von $0,37 \mu \mathrm{g} / \mathrm{m}^{3}$ gegeben.

\section{Referenzwert und vorläufiger Leitwert}

Aufgrund der großen Datenbasis mit mehr als 2000 Einzelmessungen wird das 95. Perzentil der Messdaten der AGÖF für den Referenzwert verwendet. Der Referenzwert liegt somit bei $1 \mu \mathrm{g} / \mathrm{m}^{3}$ bzw. $0,001 \mathrm{mg} / \mathrm{m}^{3}$, eine Konzentration, die mit einem Krebsrisiko von ca. $2,7 \times 10^{-6}$ verknüpft ist.

Gemäß dem AIR-Schema zur Bewertung von Kanzerogenen in der Innenraumluft wird der Referenzwert von $1 \mu \mathrm{g} /$ $\mathrm{m}^{3}$ als vorläufiger Leitwert für 1,2-DCE festgelegt [18].

\section{Hinweise zur Messstrategie}

Der vorläufige Leitwert für 1,2-DCE wurde auf Basis der VOC-Datenbank II der AGÖF abgeleitet, so dass zur vergleichenden Beurteilung von 1,2-DCE auch die Probenahmestrategie zu verwenden ist, die bei der Erstellung des Datenkollektives zu Grunde gelegt wurde (Kurzzeitmessung unter Ausgleichsbedingungen).

Wird im Rahmen einer Kurzzeitmessung der Leitwert überschritten, ist mit ei-

Bundesgesundheitsbl 2019-62:114-117 https://doi.org/10.1007/s00103-018-2849-1

(c) Springer-Verlag GmbH Deutschland, ein Teil von Springer Nature 2018

\section{Gesundheitliche Bewertung von 1,2-Dichlorethan (1,2-DCE) in der Innenraumluft}

\section{Zusammenfassung}

1,2-Dichlorethan ist im europäischen Gefahrstoffrecht als wahrscheinlich krebserzeugend beim Menschen eingestuft. Für die Risikoquantifizierung verwendeten sowohl der Ausschuss für Gefahrstoffe (AGS) als auch der Ausschuss für Risikobewertung der Europäischen Chemikalienbehörde (ECHA-RAC) die dosisabhängige Tumoranzahl in den Brustdrüsen weiblicher Ratten. Aufgrund der Unsicherheiten bei der Bewertung des gentoxischen Potentials, nutzten beide Ausschüsse für die Extrapolation in den Niedrigdosisbereich das lineare Modell. Dieses Bewertungskonzept hat auch der AIR angewendet und für ein Lebenszeitrisiko von $10^{-6}$ eine Konzentration von $0,00037 \mathrm{mg} / \mathrm{m}^{3}$ berechnet. Da der aktuelle Referenzwert (95. Perzentil gemessener Innenraumluftkonzentrationen) mit $0,001 \mathrm{mg} / \mathrm{m}^{3}$ höher liegt, wird der Referenzwert als vorläufiger Leitwert festgelegt.

\section{Schlüsselwörter}

1,2-Dichlorethan · Innenraumluft · dosisabhängige Brustdrüsen-Tumore in Ratten · Risikobewertung · Vorläufiger und risikobezogener Leitwert

\section{Health evaluation of 1,2-dichloroethane (1,2-DCE) in indoor air}

\section{Abstract}

In the European Hazardous Substances Regulation No 1272/2008 1,2-dichloroethane has been classified as probable human carcinogen. For risk quantification, the German Committee on Hazardous Substances as well as the Risk Assessment Committee of the European Chemicals Agency (ECHA-RAC) used the dose-related incidences of mammary tumours in female rats. Due to uncertainties in addressing the genotoxic potential, both committees applied the linear model for extrapolation to lower concentrations. The
German Committee on Indoor Guide Values applied the same evaluation concept and calculated a concentration of $0.00037 \mathrm{mg} /$ $\mathrm{m}^{3}$ for a life-time risk of $10^{-6}$. As the current reference value (95th of indoor air concentrations) is higher $\left(0.001 \mathrm{mg} / \mathrm{m}^{3}\right)$, the reference value is established as preliminary guideline.

Keywords

1,2-dichloroethane ·Indoor air · Dose-related mammary tumour in rats - Risk evaluation . Preliminary and risk-related guideline ner Wiederholungsmessung zu klären, ob es sich um eine einmalige oder andauernde Innenraumluftkontamination handelt.

Ergibt auch die Wiederholungsmessung eine Überschreitung des Leitwertes, sollten die Quelle sowie deren Emissionscharakteristik bestimmt werden. Für den Fall, dass von einer dauerhaft erhöhten Exposition gegenüber 1,2-DCE auszugehen ist, sind ggf. expositionsmindernde Maßnahmen einzuleiten, deren Wirksamkeit zu überprüfen ist.

\section{Anmerkungen}

Der Entwurf dieser Mitteilung wurde von Dr. Katrin Schröder, Herrn Thomas Lahrz, Herrn Herbert Grams und Dr. Martin Kraft erstellt und vom AIR im Mai 2018 verabschiedet.

\section{Literatur}

1. Verordnung (EG) Nr. $1272 / 2008$ des Europäischen Parlaments und des Rates vom 16. Dezember 2008 über die Einstufung, Kennzeichnung und Verpackung von Stoffen und Gemischen, zur Änderung und Aufhebung der Richtlinien 67/548/EWG und 1999/45/EG und zur Änderung der Verordnung (EG) Nr. 1907/2006. ABI. L 353 vom 31. Dez. 2008

2. ECHA-Datenbank, Verzeichnis der zulassungspflichtigen Stoffe. https://echa.europa.eu/de/ authorisation-list. Zugegriffen: 26. Okt. 2018

3. ECHA-RAC (2015) Application for Authorisation: Establishing a Reference Dose Response Relationship for Carcinogenicity of 1,2-Dichlorethane, RAC/33/2015/09. https://echa.europa. eu/documents/10162/13641/rac_33_dose_ response+_1_2dichloroethane_en.pdf.ZZugegriffen: 26. Okt. 2018

4. AGS (2015) TRGS 910. Begründung zu 1,2-Dichlorethan in TRGS 910 (Fassung vom 25.9.2015). https://www.baua.de/DE/Angebote/ Rechtstexte-und-Technische-Regeln/Regelwerk/ TRGS/pdf/910/910-1-2-Dichlorethan.pdf? 
blob=publicationFile\&v=2. Zugegriffen: 11 . Juni 2018

5. Health Canada (2014) Guidelines for Canadian Drinking Water Quality: Guideline Technical Document - 1,2-Dichloroethane. Water and Air Quality Bureau, Healthy Environments and Consumer Safety Branch, Health Canada, Ottawa, Ontario. (Catalogue No H144-13/3-2013EPDF). https://www.canada.ca/content/dam/canada/ health-canada/migration/healthy-canadians/publications/healthy-living-vie-saine/water-dichloroethane-eau/alt/water-dichloroethane-eau-eng.pdf. Zugegriffen: 28. Okt. 2016

6. Agency for Toxic Substances and Disease Registry (2001) Toxicological Profile for 1,2-Dichloroethane. http://www.atsdr.cdc.gov/toxprofiles/ tp.asp?id=592\&tid=110. Zugegriffen: 3 . Juni 2016

7. Institut für Arbeitsschutz der Deutschen Gesetzlichen Unfallversicherung GESTIS-Stoffdatenbank. http://www.dguv.de/ifa/gestis/gestis-stoffdatenbank/index.jsp. Zugegriffen: 28. Nov. 2016

8. EU (2017) Summary of European Commission Decisions on authorisations for the placing on the market for the use and/or for use of substances listed in Annex XIV to Regulation (EC). https://eurlex.europa.eu/legal-content/EN/TXT/?uri=uriser v:OJ.C_.2017.072.01.0002.01.ENG (No 1907/2006 of the European Parliament and of the Council concerning the Registration, Evaluation, Authorisation and Restriction of Chemicals (REACH), C/2017/1332, Document 52017XC0308(01))

9. ECHA-Datenbank, Registrierungsdossier für 1,2 Dichlorethan. https://echa.europa.eu/registrationdossier/-/registered-dossier/15430. Zugegriffen: 26. Okt. 2018

10. AGÖF (2014) Zielkonflikt energieeffiziente Bauweise und gute Raumluftqualität - Datenerhebung für flüchtige organische Verbindungen in der Innenraumluft von Wohn- und Bürogebäuden (Lösungswege). https://www.agoef.de/forschung/ fue-Il-voc-datenerhebung/abschlussbericht.html. Zugegriffen: 12. Nov. 2018

11. Chin JY, Godwin C, Parker E, Robins T, Lewis T, Harbin P, Batterman S (2014) Levels and sources of volatile organic compounds in homes of children with asthma. Indoor Air 24(4):403-415

12. Withey JR, Karpinski K (1985) The fetal distribution of some aliphatic chlorinated hydrocarbons in the rat after vapor phase exposure. Biol Res Pregnancy Perinatol 6:79-88

13. Reitz RH, Fox TR, Ramsey JC, Quast JF, Langvardt PW, Watanabe PG (1982) Pharmacokinetics and macromolecular interactions of ethylene dichloride in rats after inhalation or gavage. Toxicol Appl Pharmacol 62:190-204

14. Gwinn MR, Johns DO, Bateson TF, Guyton KZ (2011) A review of the genotoxicity of 1,2-dichloroethane (EDC). Mutat Res 727:42-53

15. Gargas ML, Clewell HJ, Andersen ME (1986) Metabolism of inhaled dihalomethanes in vivo: Differentiation of kinetic constants for two independent pathways. Toxicol Appl Pharmacol 82:211-223

16. D'Souza R, Francis WR, Bruce RD, Andersen ME (1987) Physiologically based pharmacokinetic model for ethylene dichloride and its application in risk assessment. Pharmacokinet Risk Assessment: Drink Water Health 8:286-301 (NRC, Washington DC)

17. Nagano $K$, Umeda $Y$, Senoh $H$, Gotoh $K$, Arito $H$, Yamamoto S, Matsushima T (2006) Carcinogenicity and chronic toxicity in rats and mice exposed by inhalation to 1,2-dichloroethane for two years. J Occup Health 48:424-436

18. Ausschuss für Innenraumrichtwerte (2015) Gesundheitliche Bewertung krebserzeugender Verunreinigungen der Innenraumluft - erste Ergänzung zum Basisschema. Bundesgesundheitsblatt 58:769-773 